\title{
Is Obesity a Public Health Problem?
}

\author{
Jonny Anomaly*, Duke University, NC, USA
}

*Corresponding author: Jonny Anomaly, 326 Perkins, Box 90204; Duke University; Durham, NC 27708, USA. Email: jonathan.anomaly@duke.edu.

It is often claimed that there is an obesity epidemic in affluent countries, and that obesity is one of the most serious public health problems in the developed world. I will argue that obesity is not an 'epidemic' in any useful sense of the word, and that classifying it as a public health problem requires us to make fairly controversial moral and empirical assumptions. While epidemiological evidence suggests that the prevalence of obesity is on the rise and can lead to serious health problems ranging from diabetes to cardiovascular disease, this does not by itself show that obesity is a public health problem.

Suppose you live in a small town in a remote region. You have access to outdoor recreation, but you choose to spend your leisure time watching satellite television and snacking on fried foods rather than jogging and eating oatmeal. Now suppose many other people in your town make similar choices, and that, over time, some of these people gain a lot of weight, become less productive workers and live shorter and less healthy lives than those who spend more time exercising and eating well. ${ }^{1}$ Should we consider watching television, eating junk food and becoming obese a public health problem analogous to the spread of typhus or tuberculosis? Should the government use its coercive power to change the behavior of people who make poor health choices? Does the state have the right, even the obligation, to try to make its citizens live maximally happy, healthy and productive lives?

An increasing number of scholars and journalists answer yes to some or all of these questions. Among them, some argue that obese people would prefer to live healthier lives but that they lack self-control or are victims of fast-food advertisements and a culture that normalizes unhealthy choices. On this view, anti-obesity policies would help people help themselves. Others argue that since the medical costs of obesity are absorbed by all taxpayers, workers or members of an insurance pool, extensive government intervention is justified to change people's eating and exercise behavior. There is, on this view, a public good associated with altering people's diet and exercise patterns. Both of these views are often invoked by people who consider obesity a public health problem. The first view sees public health measures as a set of paternalistic policies that help people live healthier lives. The second view sees public health as the provision of health-related public goods-in this case, the reduction of social costs associated with obesity. ${ }^{2}$
I will argue that although public health should primarily be concerned with the provision of public goods (Epstein, 2004; Anomaly, 2011) and that reducing obesity may constitute a public good under certain circumstances, obesity should not necessarily be regarded as a public health problem. This is true for at least two reasons, which I will mention briefly and then discuss in more detail in the body of the article.

First, reducing obesity is only a public good over the long term if the health costs and productivity losses associated with obesity are borne by all taxpayers, workers or members of an insurance pool, and if obese people live long enough to extract more resources than they contribute in taxes, work productivity or insurance premiums.

Second, not all health-related public goods should be on the public health agenda since some public goods are an artifact of morally contentious social policies. For example, we normally think of improvements in diet and exercise as a private good, because each person who eats well and exercises regularly enjoys better health. However, if government policies compel healthy people to cover the costs of obese people, improvements in exercise and eating habits become a pure public good—one that benefits all taxpayers, workers, or members of an insurance pool. When public policy spreads the cost of risky behavior, we essentially become partial stakeholders in the (otherwise personal) decisions of others: we suffer when they are unhealthy and unproductive, and we benefit when they are healthy and productive. Whether such policies are desirable, however, has been fiercely contested (Boaz, 2004; Balko, 2005). Because government policies can transform private goods into public goods by spreading the costs and benefits of each person's behavior, whether we should regard obesity as a problem of public health or private health may ultimately depend on the moral justification 
for health-care policies that determine who pays for obesity. $^{3}$

\section{Cultural Contagion}

I want to begin by considering the language in which the obesity debate is often framed. Many anti-obesity activists claim that obesity is an 'epidemic' or even a 'pandemic' because its prevalence is increasing at a rate comparable to the spread of infectious diseases (Swinburn et al., 2011). According to Oxford Dictionaries Online, an epidemic is a 'widespread occurrence of an infectious disease in a community at a particular time'. Some authors have begun to use the term more loosely to include anything that negatively impacts the health of a large number of people in a population, and many dictionaries have begun listing this as a secondary definition. ${ }^{4}$ But this definition is so vague as to include popular but dangerous activities like playing ice hockey in Montreal, wearing high heels in Paris and driving an automobile in Delhi.

It's true, of course, that becoming obese, playing ice hockey, wearing high heels and driving a car all put one at risk of acquiring a chronic disease $\mathrm{e}^{5}$ or otherwise impairing one's health or shortening one's life. Heels hamper one's ability to run from an attacker and increase the chance that one will incur a twisted ankle or permanent joint damage. They are expensive and dangerous, and their increasing popularity is testament to the power of evolutionary arms races in sexual selection. But although the trend of buying and wearing high-heeled shoes meets the more expansive definition mentioned above, few people would describe it as an epidemic even if all women would be better off if high heels were never invented. For similar reasons, it is a stretch to describe the dietary and exercise habits that lead to obesity (or obesity itself) as an epidemic, even if many obese people would be better off had deep-fried foods and satellite television never been invented. When physicians, scientists and public health activists claim that ' $\mathrm{X}$ is an epidemic', the words conjure images of a rapidly spreading infectious disease that claims victims indiscriminately, not people driving on a highway, strutting around in stilettos or consuming too many carbohydrates.

Perhaps we can more charitably interpret the popular use of words like 'epidemic' or 'pandemic' to describe the increasing prevalence of obesity. These words might be used metaphorically to describe a medical affliction that uses culture as a vector. Smoking is an example of what we might call 'cultural contagion'. When a critical mass of people, especially influential celebrities, smoke cigarettes in public, it may be more likely that other people will feel pressure to smoke, even if they would prefer to live in a culture in which nobody smoked (Goodin, 1990; Verweij, 2007). Similarly, as the number of severely overweight people in a population rises, perhaps obesity becomes normalized so that people who would otherwise feel social pressure to stay fit no longer do.

Although I find this argument plausible in the case of smoking and there may be some truth to the assertion that your probability of becoming obese increases when others around you are obese (Christakis and Fowler, 2007), there is a significant disanalogy between obesity and smoking. First, while men often find curvy women attractive (provided they have a roughly 0.7 waist-to-hips ratio), obesity is not generally considered sexually attractive to men or women, and there are good reasons to believe that our tendency to regard extremely overweight people as unattractive is- to some extentbiologically hardwired (Miller, 2001). Second, there is strong evidence that people who are obese are discriminated against, perhaps because they are thought to lack self-control and self-confidence (Puhl and Brownell, 2001; Carr and Friedman, 2005; Puhl, 2011). ${ }^{6}$ This is generally not true with smoking, which (at one time, at least) was considered glamorous, especially among young people (Potts et al., 1986). ${ }^{7}$ These considerations suggest that obesity is not culturally contagious in the same way that smoking was in the 20th century. As with any other social trend, people can be impacted by the choices of others (some of us will see obese people and lose our desire to lose weight, others will see them and have a renewed sense of why we need to stay in good shape), but we are not 'infected' with obesity in the unpredictable and involuntary way in which we can be infected with influenza or Ebola.

Maybe those who advocate urgent collective action to control obesity—especially government action — tend to use words like 'epidemic' and 'public health crisis' because infectious disease is widely considered a powerful justification for state action. All reasonable people agree that promoting vaccination and improving sanitation may merit the restriction of individual liberty to achieve goals which we lack the power to unilaterally produce (for example, an environment free of Measles). ${ }^{8}$ Invoking the language of contagion when describing obesity is therefore likely to get people's attention and garner public support.

David Boaz (2004) has emphasized the role of language in framing the policy debate: "Language matters. Calling something a 'public health problem' suggests 
that it is different from a personal health problem in ways that demand collective action. And while it doesn't strictly follow, either in principle or historically, that 'collective action' must be state action, that distinction is easily elided in the face of a 'public health crisis'." A similar point can be made about the widespread use of words like 'epidemic' and 'pandemic' to describe the problem of obesity. We are free to use words any way we like, provided that we clearly indicate what we mean. But we should resist the temptation to use hyperbolic language simply to gain sympathy for politically controversial goals.

\section{The Cost of Obesity}

A common argument for treating obesity as a public health problem appeals to the social costs that obese people are said to impose on others. The two most relevant costs are the financial burden obese people may impose on other taxpayers or members of an insurance pool, and decreased average productivity among obese workers (Averett, 2011; Cawley, 2011; Finkelstein and Yang, 2011).

The issue of external costs is a little more complicated, however, than some have suggested. For one thing, it is important to distinguish the annual costs of obesity from lifetime costs. Although obese people generally impose a higher burden on shared medical resources in any given year, it is conceivable that because obese people live shorter lives, they contribute more than they take away from common medical resources over a lifetime (Finkelstein and Yang, 2011). There is now fairly good evidence that this is true for smokers (Barendregt et al., 1997: 1052-1057), and at least one study in Holland suggests the same is true for obesity (van Ball et al., 2008: 242-249). If it turns out to be true that obese people provide a net social benefit for shared medical resources, the argument from social cost fails. In fact, it may be used to justify government subsidies rather than taxes for cigarettes and junk food.

Another complicating factor is that most studies of the cost of obesity take current policies as fixed parameters, and then ask where we should go from here, assuming these policies will remain in place. But if we're ultimately interested in crafting more fair and cost-effective policy, we should also ask fundamental questions, including whether existing mandates fairly distribute medical costs and whether they increase or decrease moral hazard.
Moral hazard occurs when the costs of risky behavior are shared, but the benefits go to the individual people taking risks. In the case of obesity, some people probably pay less attention to the medical consequences of their diet and exercise routines because they bear only a small fraction of the financial costs they incur as a result (Finkelstein and Zuckerman, 2008). If we allowed insurance companies to charge higher premiums or airlines to charge higher prices for heavier people, for example, there might be a greater incentive for obese people to lose weight and for the rest of us to guard against becoming obese. Taxing obesogenic foods could have the same effect, but the taxes would have to be quite high and would be borne (arguably unfairly) by non-obese people as well. I am not arguing for or against such taxes, but rather pointing out that policies can create moral hazard, which increases socially costly behavior by removing the natural incentives people have to take care of their own health.

Another problem for the social cost argument is that some researchers include lower productivity at work and lower expected wages (presumably because of lower productivity) as social costs of obesity (Wyatt et al., 2006). However, these should be regarded primarily as private costs. One person's wage doesn't negatively affect another (more productive) person's wage except in the very indirect way that each person's wage has a marginal impact on the average wage of others in her field (and this effect can be positive or negative). Moreover, workers are unproductive for many different reasons-including stress, insomnia and compulsive internet use-and unless we think governments should try to make workers maximally productive, it's not clear that they should pay special attention to obesity. $^{9}$

Obesity reduction is often said to be an important public health goal because there is a public good associated with reducing obesity, since all taxpayers, workers or members of an insurance pool share the benefits that come with fewer fat people in the overall population. What I have argued is that this claim is not necessarily true when we consider the costs and benefits over long periods of time, and that even if it is true, it may be due in part to unfair or otherwise undesirable government policies that lead people to ignore the costs of imprudent behavior. This is not necessarily an argument against all anti-obesity efforts (especially those aimed at children). It is instead a call for skepticism about the extent to which obesity should be regarded as a public health problem. 


\section{Public Health and Public Policy}

In the introduction, I mentioned that some public goods problems are created by morally controversial social policies rather than being a fundamental feature of the human condition. Policies that socialize the costs of obesity may be an example: once such policies are in place, we each have powerful reasons to regulate other people's behavior.

A clearer, and related, example involves government subsidies and price controls for agricultural commodities. The American government (like many other governments) gives large subsidies to farmers who produce staple crops like corn and wheat. Many have noted that these subsidies artificially lower the price of unhealthy carbohydrates and even create an incentive to raise and feed cattle in a way that makes their meat less healthy for consumers (since cattle evolved to eat grass rather than grains). Worse still, food subsidies distort markets, undermine mutually beneficial free trade agreements and cost taxpayers money. They are a paradigm of unfair and inefficient public policy.

Some anti-obesity activists have suggested that, to counteract subsidies for grains, governments should subsidize fruits and vegetables, or impose new price controls for healthy foods, so that the relative price of obesogenic foods like cookies and candy increases (Swinburn et al., 2011; Institute of Medicine, 2012). Once subsidies for carbohydrate-rich foods are in place, of course, there is an argument for leveling the playing field by increasing subsidies for other foods, since none of us wants to be penalized for making healthy choices. But rather than justifying subsidies that further distort markets and unfairly reward particular producers at the expense of consumers, this argument suggests that we should eliminate subsidies for all foods. Moreover, if, as some suggest, subsidies for staple crops like corn have had a negligible impact on obesity rates (Alston et al., 2008), subsidies for healthy foods would have to be much larger than the already massive payments to corn and wheat farmers to have any real impact on obesity. This would exacerbate existing inefficiencies in agricultural markets and would invite even more lobbying of government officials by farmers and food producers.

The argument that we should increase subsidies for healthy foods to decrease obesity shows how one bad policy (government subsidies for grains) can transform what would otherwise be another bad policy (government subsidies for vegetables) into a relatively pure public good. But in this case, it is more sensible to remove the initial policy rather than stir another expensive policy into the already costly cauldron. In the same way, it could be argued that some of the policies that socialize the cost of obesity and thereby make obesity reduction a public good should be changed rather than used to support further government policies that spread the costs even more.

I have not argued that all anti-obesity measures are unjustified. I have instead suggested that the public goods argument for reducing obesity is not very convincing, and that obesity is therefore not necessarily a public health problem. There may be other reasons to enact anti-obesity policies. For example, some think mildly paternalistic policies that nudge people toward healthier choices are worth exploring — perhaps because they would agree to such measures if they were fully rational (Thaler and Sunstein, 2003: 1159-1202). ${ }^{10}$ Less controversially, we might have a variety of reasons to improve nutrition education for children, to ban unhealthy foods at schools and to encourage exercise for adolescents. These policies protect children from making myopic choices that harm them later on. But applying such programs to adults is far more controversial for familiar reasons, and we should remain cautious about considering such programs a core part of the public health agenda. An overly expansive conception of public health risks alienating public health allies who oppose paternalistic policies or who are skeptical of policies that incentivize each of us to take an interest in shaping how other people live their lives.

\section{Conflicts of interest}

None declared.

\section{Notes}

1. Two provisos are in order. First, although many who support anti-obesity programs emphasize the need to change the urban environment, the rural Southern regions of the United States have greater rates of obesity than many urban parts of the United States (Levi et al., 2011). Second, I will assume that in most cases behavior that contributes to obesity is at least partially voluntary. While I readily acknowledge that genetic and social determinants can help explain obesity, I am not convinced, and it is not possible to use science to show that most cases of obesity are fully causally determined by environmental and physiological facts. The reason it is not possible to show this is that the denial of free will is a 
metaphysical thesis that cannot be settled by empirical findings. There is a temptation to think that explaining the origin of a condition or action shows that choice played no part in creating it. But if this is true, then all explanations of action that appeal to biochemistry and social determination would undermine voluntary choice. This raises deep questions about determinism. For the purpose of this article, I will set aside these questions and assume that in most cases weight gain and loss is at least partially under our control.

2. I should note that a good is public, in the technical sense, if it exhibits two features: non-rivalry and non-excludability. Non-rivalry (or 'jointness in consumption') indicates that one person's consumption of a good leaves an equal amount for others, while non-excludability occurs when nobody can be excluded from enjoying a good. Herd immunity against an infectious disease is a paradigmatic public good.

3. If the bundle of policies that determine who pays for obesity is itself a relatively pure public good for which there is widespread demand, then the case could be made that certain extensions of these policies are also public goods and therefore part of public health. If not, extensions of these policies should not necessarily be regarded as public health policies.

4. For an excellent discussion of the history and controversy surrounding the term 'epidemic', see Green et al. (2002: 3-6).

5. Some activists would like organizations like the American Medical Association to classify obesity as a disease, but this is often for practical reasons-so that state governments and insurance companies will have to pay for the 'treatment' of obesity.

6. Indeed, while social disapproval may cause further health problems or psychological trauma for some obese people, for many, it may serve as a natural penalty (to paraphrase John Stuart Mill) that causes them to alter their behavior in a healthier direction.

7. In biological terms, perhaps smoking is sometimes considered attractive because it's a costly signal ('I am so fit that I can afford to take on the additional risk of smoking' or 'I am not as poor as you think: I can afford to spend extra income on cigarettes').

8. A reviewer for an earlier draft of this article suggested that it is not true that all 'reasonable' people agree that government programs to promote vaccination and sanitation are justified, because some religious people are legally entitled to opt out of such programs. I couldn't disagree more. First, the fact that laws allow religious exceptions to otherwise universal mandates for collective action says nothing about whether the mandates are justified or whether those allowed to opt out are reasonable. Second, if people believe they have a monopoly on religious truth, and that this entitles them to put other people in danger by opting out of vaccination programs, this epitomizes unreasonable behavior.

9. We might concede that when people are less productive, they generate less tax revenue for their government than those who are more productive. But if we want to take this argument to its logical conclusion, governments should also try to discourage less productive people from reproducing, because the children of such people will, on an average, produce less in tax revenue than the children of other more productive people.

10. For such policies to be justified, we would have to make several assumptions: (i) that any benefits they deliver are worth the costs to everyone of implementing the policy, (ii) that government agents will not be subject to the same biases that they are tasked with helping citizens overcome, and (iii) that government agents are not likely to abuse their power by implementing hard paternalistic policies-which override people's considered judgments about how to live their lives - by appealing to soft paternalism - which occurs when government policy is used to help people overcome their own acknowledged infirmities.

\section{References}

Alston, J., Sumner, D. and Vosti, S. (2008). Farm Subsidies and Obesity in the United States. Food Policy, 33, 470-479.

Anomaly, J. (2011). Public Health and Public Goods. Public Health Ethics, 4, 251-259.

Averett, S. (2011). Labor Market Consequences: Employment, Wages, Disability, and Absenteeism. In: The Oxford Handbook of the Social Science of Obesity. Oxford: Oxford University Press, pp. 531-552.

Balko, R. (2005). Does Obesity Justify Big Government? The Freeman, 55, available from: http://www.thefree manonline.org/features/does-obesity-justify-biggovernment/ [accessed May 2012]. 
Barendregt, J., Bonneux, L. and van der Mass, P. (1997). The Health Care Costs of Smoking. New England Journal of Medicine, 337, 1052-1057.

Boaz, D. (2004). CATO Institute: Obesity and 'Public Health', available from: http://www.cato.org/ publications/commentary/obesity-public-health [accessed 30 October 2012].

Carr, D. and Friedman, M. (2005). Is Obesity Stigmatizing? Journal of Health and Social Behavior, 46, 244-259.

Cawley, J. (2011). The Economics of Obesity. In: The Oxford Handbook of the Social Science of Obesity. Oxford: Oxford University Press, pp. 120-137.

Christakis, N. and Fowler, K. (2007). The Spread of Obesity in a Large Social Network Over 32 Years. New England Journal of Medicine, 357, 370-379.

Epstein, R. (2004). In Defense of the 'Old' Public Health. Brooklyn Law Review, 69, 1421-1470.

Finkelstein, E. and Yang, H. (2011). Obesity and Medical Costs. In: The Oxford Handbook of the Social Science of Obesity. Oxford: Oxford University Press, pp. 495-501.

Finkelstein, E. and Zuckerman, L. (2008). The Fattening of America. New Jersey: Wiley.

Goodin, R. (1990). No Smoking: The Ethical Issues. Chicago: University of Chicago Press.

Green, M., Swartz, T., Mayshar, E., Lev, B., Leventhal, A., Slater, P. and Shemer, J. (2002). When Is an Epidemic an Epidemic? Israel Medical Association Journal, 4, 3-6.

Institute of Medicine (IOM). (2012). Accelerating Progress in Obesity Prevention: Solving the Weight of the Nation, available from: http://www.iom.edu/ Reports/2012/Accelerating-Progress-in-ObesityPrevention.aspx [accessed 15 May 2012].

Levi, J., Segal, L., Laurent, R. and Kohn, D. (2011). F as in Fat: How Obesity Threatens America's Future, Trust for America's Health and Robert Johnson
Wood Foundation, available from: http://www .healthyamericans.org/assets/files/TFAH2011FasIn Fat10.pdf.

Miller, G. (2001). The Mating Mind: How Sexual Choice Shaped the Evolution of Human Nature. New York: Anchor Books.

Oxford Dictionaries Online, 'Epidemic', available from: http://oxforddictionaries.com/definition/epidemic? region=us [accessed 30 October 2012].

Potts, H., Gillies, P. and Herbert, M. (1986). Adolescent Smoking and Opinion of Cigarette Advertisements. Health Education Research, pp. 195-201.

Puhl, R. and Brownell, K. (2001). Bias, Discrimination, and Obesity. Obesity Research, 9, 788-805.

Puhl, R. (2011). Bias, Stigma, and Discrimination. In: The Oxford Handbook of the Social Science of Obesity. Oxford: Oxford University Press, pp. 553-571.

Swinburn, B., Sacks, G., Hall, K., McPherson, K., Finegood, D., Moodie, M. and Gortmaker, S. (2011). The Global Obesity Pandemic: Shaped by Global Drivers and Local Environments. The Lancet, 378, 804-814.

Thaler, R. and Sunstein, S. (2003). Libertarian Paternalism Is Not an Oxymoron. University of Chicago Law Review, 70, 1159-1202.

van Ball, P., Polder, J., de Wit, G., Hoogenveen, R., Feenstra, T., Boshuizen, H., Engelfriet, P. and Brouwer, W. (2008). Lifetime Medical Costs of Obesity. PLoS Medicine, 5, 242-249.

Verweij, M. (2007). Tobacco Discouragement: A NonPaternalistic Policy. In: Ethics, Prevention, and Public Health. Oxford: Oxford University Press, pp. 179-197.

Wyatt, S., Winters, K. and Dubbert, P. (2006). Overweight and Obesity: Prevalence, Consequences and Causes of a Growing Public Health Problem. Journal of the Medical Sciences, 331, 166-174. 